\title{
Propuesta de evaluación alfanumérica de la competencia transversal “CT-05. Diseño y Proyecto" integrada en la evaluación la asignatura Ingeniería Fluidomecánica, asociada al PIME B24
}

Modesto Pérez-Sánchez ${ }^{\mathrm{a}}$, José Vinaches Ramis ${ }^{\mathrm{a}}$, J. Ricardo Satorre Aznar, P. Amparo López-Jiménez ${ }^{\mathrm{a}}$

a Departamento de Ingeniería Hidráulica y Medio Ambiente. Universitat Politècnica de València. mopesan1@upv.es, jvinache@upvnet.upv.es, josaaz@dihma.upv.es, palopez@upv.es;

\begin{abstract}
The introduction of active methodologies inside of the teaching is contributing to analyze the learning as a continue formative procedure of the student, neglecting the master classes partially where the lack of motivation by the student can suppose a obstacule to reach the learning results of the students. The current research describes the introduction of active methodologies in different years in the subject, called "Fluid Mechanics Engineering" that is teaching in the Escuela Politècnica Superior of Alcoi. The contribution specially shows the alphanumeric evaluation of a transversal competence, called "Design and Project". This competence is currently in the evaluation exercises of the subject.
\end{abstract}

Keywords: CT-05 Design adn Project; Fluid Mechanics Engineering; Autonomus Learning; Rubbric.

\begin{abstract}
Resumen
La introducción de metodologías activas dentro de la enseñanza está contribuyendo a ver el aprendizaje como un proceso formativo continuo del alumno, abandonando parciamente las clases magistrales tradicionales donde la falta de motivación por parte del alumno podía suponer un obstáculo al alcance de los resultados de aprendizaje de los estudiantes. En la presente comunicación se describe la introducción de metodologías activas a lo largo de diferentes cursos académicos en la asignatura de Ingeniería Fluidomecánica en la Escuela Politécnica Superior de Alcoy, haciendo especial hincapié en la evaluación alfanumérica de la competencia transversal Diseño y Proyecto como parte de los actos de evaluación de la asignatura.
\end{abstract}

Palabras clave: CT-05 Diseño y Proyecto; Ingeniería Fluidomecánica; Aprendizaje permanente; Rúbrica; 


\section{Introducción}

Desde la Educación Primaria hasta la Educación Superior, es cada vez más común poner en práctica procesos de enseñanza-aprendizaje basados en la experimentación práctica en el aula. En estos modelos, el maestro es el responsable de guiar al alumno para que pueda alcanzar su nivel máximo de desarrollo potencial aprovechando las habilidades y las aptitudes de cada niño (Wood, 1976, SPM, 2008). Este progreso académico permite reducir el número de clases magistrales para comprender las materias contenidas.

En ocasiones, los métodos tradicionales pueden ser poco motivadores para los estudiantes cuando el tema desarrollado presenta un alto grado de formalismo en algunas explicaciones. Durante la clase magistral, los estudiantes tienen que esforzarse más para memorizar la información, la cual es parcialmente olvidada posteriormente (Morales, 2004). Además, estas metodologías fomentan el trabajo individual, con el consiguiente perjuicio de no trabajar la competencia de trabajo en equipo. Para evitar la falta de motivación en los estudiantes y promoverla, los estudios universitarios pueden enfocarse como una etapa de la vida donde las adquisiciones de conocimiento son necesarias para conseguir un trabajo. Por lo tanto, es necesario buscar nuevos enfoques educativos, tales como el "Aprendizaje basado en problemas" (PBL) el cual busca conseguir que los estudiantes adquieran las habilidades suficientes para poder manejar cualquiera de las situaciones complejas que pueden enfrentarse en su etapa profesional (Benavides, 2009).

Relacionado con esa capacidad de aprendizaje, Edgar Dale (1969) sugirió un modelo sobre la efectividad de los métodos de aprendizaje, realizando una comparación sobre la capacidad de retener información de una participación activa y pasiva de los estudiantes y relacionándola con la adquisición de información, así como su influencia al generalizar los aprendizajes. Esta comparación fue presentada a través de un cono de aprendizaje (Edgar, 1969; Master, 2013) que muestra la capacidad del alumno para retener (Figura 1).

(cc) EY-NC-ND 2018, Universitat Politècnica de València 


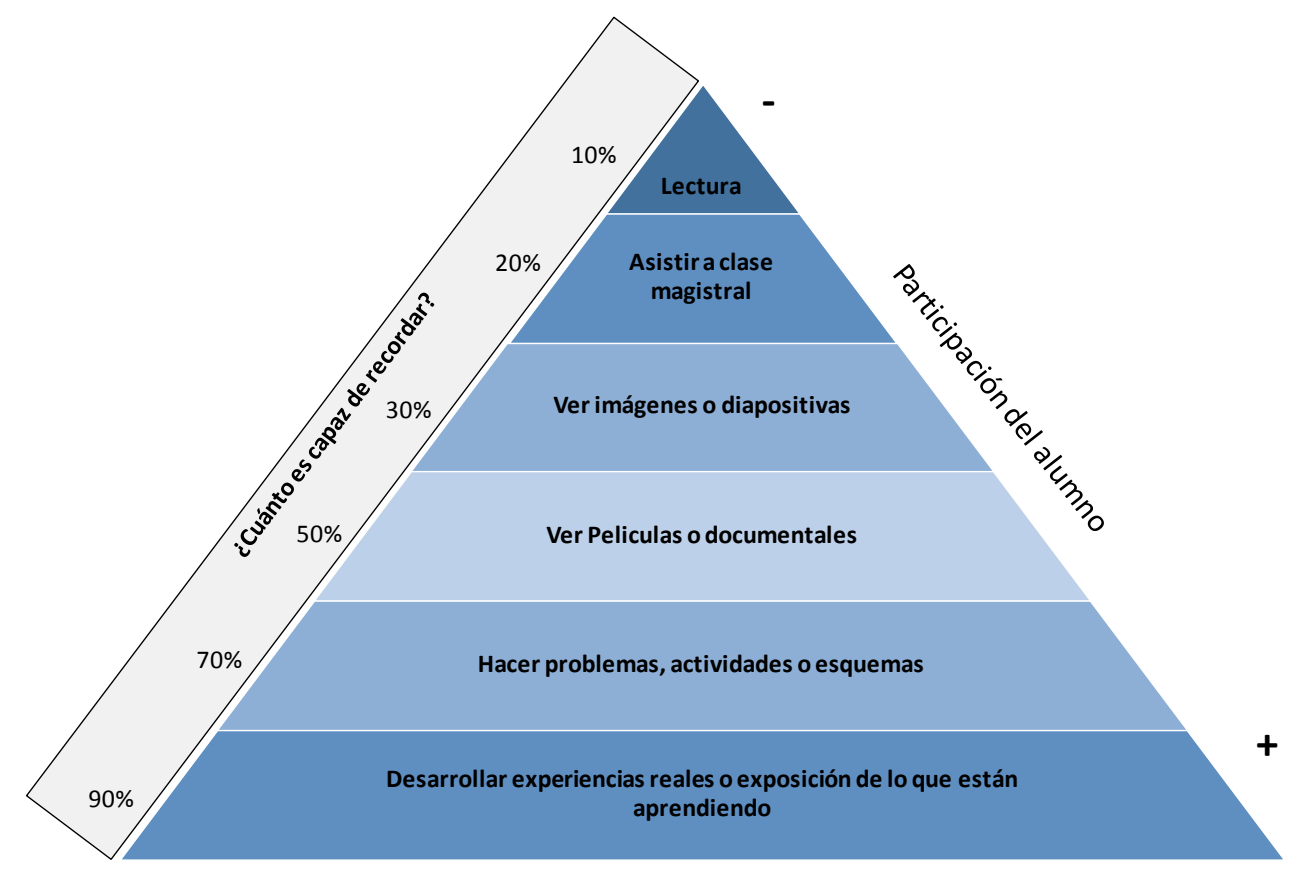

Fig 1. Metodologías vs Participación del alumno

La Figura 1 muestra el aprendizaje retenido en un alumno como función de las herramientas de enseñanza (por ejemplo, clase magistral, películas, discusión). La interacción y participación del estudiante durante el aprendizaje es crucial para promover la actitud del alumno (activo o pasivo) (Rámirez-Echeverry, 2016). Normalmente, si el estudiante solo recibe información a través de clases magistrales, clases magistrales y / o presentaciones, tiene una actitud más pasiva y el conocimiento retenido es menor (10-30\% del total de conceptos enseñados; pirámide en la Figura 1). Considerando que, si el estudiante participa en metodologías activas donde se integra en el aprendizaje (por ejemplo, resolución de problemas, experimentación, discusión), el conocimiento retenido aumenta (50-90\% del total de conceptos enseñados según la metodología educativa; parte inferior de la pirámide) en la Figura 1). La razón de esto es que PBL hace que el alumno sea responsable y una parte activa en todo su proceso de aprendizaje. En estas metodologías activas, el profesor es visto como un facilitador del proceso de enseñanza-aprendizaje, que da al alumno la oportunidad de aprender a aprender y desarrollar sus habilidades cognitivas y habilidades de cooperación como miembro de un equipo.

\section{Objetivos}

El objetivo de la presente comunicación es, por un lado, mostrar la evolución relativa a la propuesta de evaluación llevada a cabo en la asignatura Ingeniería Fluidomecánica (Iti. 2) de tercer curso del Grado de Ingeniería Mecánica de la Escuela Politécnica Superior de Alcoy por docentes del Departamento de Ingeniería Hidráulica y Medio Ambiente. Por otro 
lado, mostrar la evaluación alfanumérica relativa a la competencia transversal "Diseño y Proyecto” La asignatura de Ingeniería Fluidomecánica es troncal, se imparte en tercer curso de cuatrimestre $\mathrm{B}$, tiene una carga docente de 6 créditos y una extensión no presencial estimada de $90 \mathrm{~h}$. La asignatura se imparte una vez el alumno ha cursado los dos primeros cursos de grado, donde ha integrado dentro de su conocimiento los conceptos fundamentales de asignaturas básicas ingenieriles como son matemáticas, física, resistencia de materiales y mecánica de fluidos, entre otras. En esta situación, el alumno está cerca de escoger la mención de Grado, por lo que su visión ingenieril comienza a estar "sesgada" por los gustos o vocación del alumno. Este hecho, es importante, ya que hace que la asignatura tenga que ser atractiva ya que puede existir un gran número de alumnos que no estén interesados a priori por la asignatura ya que no la ven relacionada con la mención que consideran escoger (Diseño e Ingeniería de Vehículos, Diseño y Cálculo Materiales Poliméricos y Compuestos; Diseño y Fabricación Máquinas y Prototipos, Ingeniería de Proyectos). Aunque las máquinas hidráulicas esta presenta en todas ellas, solo los alumnos que tienen claro que cursaran la mención de Ingeniería de Proyectos ven utilidad en la asignatura, por lo que la motivación por parte del personal docente que interviene en esta materia es crucial.

Por lo tanto, desde el departamento, así como desde los docentes implicados, el hecho de cursar Ingeniería Fluidomecánica, debe conseguir alcanzar los resultado de aprendizaje establecidos en las competencias generales y específicas del título, mediante el desarrollo de estrategias que integren las competencias transversales que trabaje o sea punto de control (evalue) en Dominio II. Estas estrategias estarán diseñadas de forma que:

1. No suponga una carga de trabajo extra por los alumnos

2. Motive al alumno a llevar al día la asignatura y, por tanto, la evaluación continua cobre importancia y realismo. Esta evaluación debe ayudar al alumno a sentirse apoyado durante las $168 \mathrm{~h}$ (presencial y no presencial) de trabajo que recoge la guía docente.

3. El alumno vea la aplicabilidad de la asignatura en cualquier campo que esté presente, ya sea una bomba de engranajes recirculación de aceite de un motor, una turbina a instalar en un pequeño azud o diseño de un rodete específico para bombear un fluido con características especiales en una industria.

4. Permitan evaluar la competencia transversal que la asignatura es punto de control. Este curso 2016/2017 fue en Análisis y Resolución de Problemas, Diseño y Proyecto, Responsabilidad Social, Ética y Medio Ambiental, así como Aprendizaje Permanente.

Dentro de la presente comunicación, se centrara en la propuesta metodológica y resultados alcanzados en el curso 2016/2017 en la asignatura, teniendo en cuenta la competencia transversal Diseño y Proyecto. Del mismo modo, se presenta el desarrollo para este curso 2017/2018 de una rúbrica alfanumérica que permita la evaluación de la competencia tanto con números como con letras.

El desarrollo de esta rúbrica se encuentra dentro del Proyecto de Innovación y Mejora Docente (PIME) "Desarrollo de Metodologías de Evaluación y Diseño de Rúbricas de la Competencia Transversal (CT) 05 Diseño y Proyecto aplicado al campo de la Ingeniería

(cc) EY-NC-ND 2018, Universitat Politècnica de València 
Hidráulica (Fase II), el cual se encuentra integrado el Equipo de Innovación y Calidad Educativa (EICE) denominado Desarrollo de Metodologías Activas de evaluación aplicadas al campo de la Ingeniería Hidráulica (DESMAHIA).

\section{Desarrollo de la innovación docente}

\section{Evolución de la planificación de la asignatura relativa a la innovación docente}

La innovación docente llevada a cabo en la asignatura esta implantándose parcialmente por etapas desde el curso 2014/2015, donde se detectaron carencias motivacionales por parte del alumno, en el cual, el $65 \%$ no vea utilidad práctica a la asignatura, un $28 \%$ la consideraba útil y sólo un 7\% consideraba que la asignatura era muy útil para su formación. Este hecho, unido a la falta de demanda de TFGs por parte de los alumnos, síntoma que mostraba la falta de motivación por parte de los alumnos hacia esta asignatura.

Por ello, se estableció una implantación de metodologías activas que ayudaran a fomentar el trabajo autónomo del alumno, las cuales se han ido integrando poco a poco dentro de la asignatura que conllevará su finalización para el curso 2019/2020. La implantación de las metodologías vinieron acompañadas por el desarrollo del Plan Estratégico de la Universitat Politècnica de València (UPV), por lo que la implantación de la evaluación de las competencias transversales ha ido de forma paralela. De forma breve, el desarrollo temporal de las diferentes metodologías ha sido:

- $\quad$ Curso 2015/2016. Introducción de un proyecto de dimensionado relacionado con los contenidos teóricos impartidos, dónde el alumno pudiera relacionar la teoría con aplicaciones prácticas de la realidad. Este trabajo académico, donde el estudiante recibe los conceptos teóricos y uso de software mediante clase presencial, es desarrollado de forma no presencial y tiene un peso específico del $20 \%$ en la evaluación de la asignatura.

- $\quad$ Curso 2016/2017. Considerando el Plan Estratégico UPV, donde la asignatura es punto de control en nivel de dominio II de las competencias CT-03 'Análisis y Resolución de Problemas”, CT-07 'Responsabilidad Social, Ética y Medio Ambiental' y CT-11 'Aprendizaje Permanente', se plantea la estrategia de propuesta de tareas por revisión por pares, la cual fue descrita en Pérez-Sánchez et al., (2017b) para la evaluación de la CT-03. La incorporación de esta estrategia, supuso el incremento en la tasa de rendimiento de la asignatura, el incremento de las tutorías lo que indicaba que los alumnos llevaban una asimilación de los conceptos progresiva y continua a medida que se impartía la docencia. Al mismo tiempo se introdujeron actividades a través de PoliformaT y presenciales donde se llevaba a cabo el empleo de conceptos técnicos aplicados de la asignatura al campo aplicado de la responsabilidad social, ética y medioambiental, utilizando el debate como una de las herramientas de evaluación (Pérez-Sánchez y LópezJiménez, 2017a). Integrada en el PIME "Desarrollo de Metodologías de Evaluación y Diseño de Rúbricas de la CT-05 Diseño y Proyecto aplicado al 
campo de la Ingeniería Hidráulica”, donde el trabajo académico (proyecto) se integra para el trabajo y evaluación de la competencia transversal.

- $\quad$ Curso 2017/2018. La asignatura mantiene las todas las estrategias de aprendizaje integradas en el curso anterior, intensificando el uso de las tareas revisadas por pares e integrando la competencia transversal CT-05 dentro de la evaluación de la asignatura al evaluar numéricamente los descriptores de cada uno de los indicadores. El tratamiento de los descriptores de forma numérica y alfabética, es fruto de las reuniones mantenidas con responsables de seguimiento de la Comisión de Evaluación y Seguimiento de Proyectos de Innovación y Mejora Educativa CESPIME e Instituto de Ciencias de la Educación (ICE) del PIME actual en progreso. Durante este curso, al igual que el anterior se introduce el Proyecto Docencia en Red, donde se desarrollan un total de 23 Polimedias específicos que pueden ser usados en esta asignatura.

- $\quad$ Curso 2018/2019. Para el siguiente curso, desde el personal docente que imparte la asignatura, se optará por la integración de las tareas dentro de la evaluación de la asignatura, eliminando actos de evaluación presenciales (tradicionales exámenes y/o parciales) y sustituyéndolos parcialmente por evaluación de las tareas.

\section{Metodología desarrollada para la competencia Diseño y Proyecto en la asignatura Ingeniería Fluidomecánica}

La planificación llevada a cabo por parte del profesorado que lleva a cabo el desarrollo de la asignatura, está compuesta por 5 fases diferentes (Figura 2), las cuales se encuentran integradas dentro del PIME y son semejantes a todas ellas.

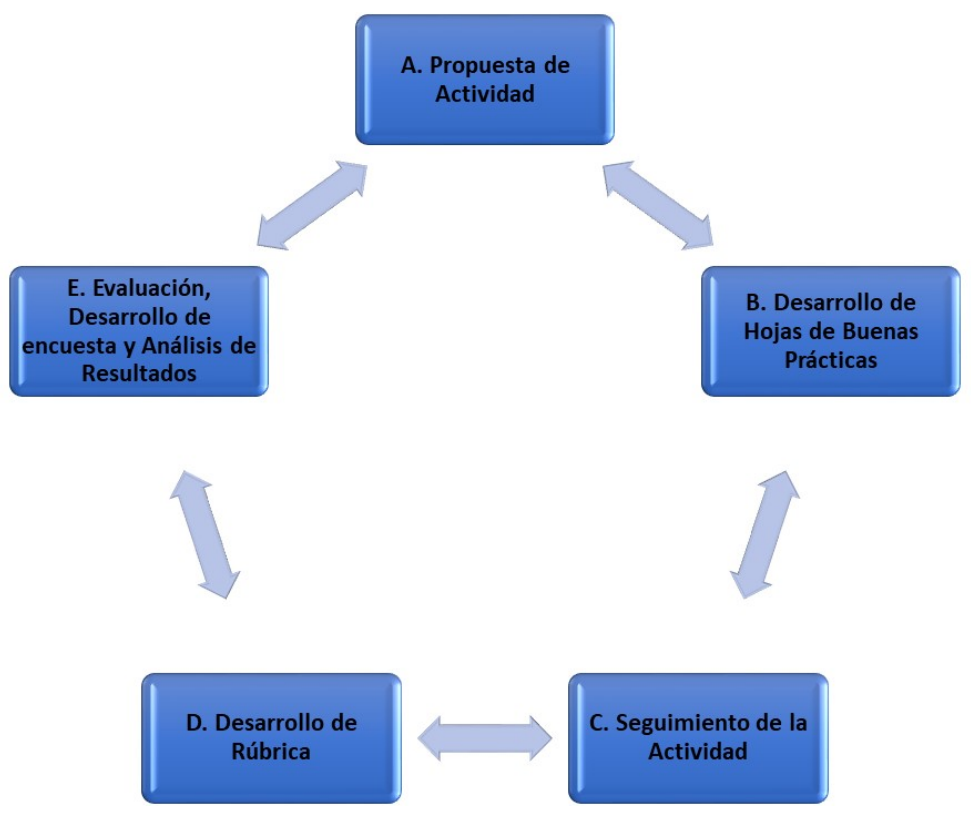

Fig 2. Planificación de la Actividad propuesta en Ingeniería Fluidomecánica

(cc) EY-NC-ND 2018, Universitat Politècnica de València

Congreso IN-RED (2018) 
A. Propuesta justificada de actividad, considerando en este caso concreto de la asignatura Ingeniería Fluidomecánica, está relacionado con la selección de bombas aplicadas a sistemas de distribución.

B. Desarrollo de la hoja de buenas prácticas de cada una de las actividades a desarrollar. Esta hoja de buenas prácticas incluye la totalidad de las actividades involucradas dentro de la asignatura. En cada actividad, se definen los resultados de aprendizaje en dicha actividad, así como para la competencia en concreto. Se elabora la estrategia de evaluación, estableciendo tipo de evaluación, peso, instrumento de evaluación, así como profesores que llevan a cabo la evaluación. En el caso concreto de la asignatura de Ingeniería Fluidomecánica, las competencias CT-03 y CT-07 utilizan rúbricas de evaluación las cuales fueron desarrolladas (Pérez-Sánchez y López-Jiménez, 2017a; Pérez-Sánchez et al., 2017b)

C. Seguimiento de la actividad. En este caso, los docentes involucrados contribuyen a dar apoyo al estudiante tanto en las fases presenciales como no presenciales. Es en este punto donde las tutorías y Polimedias, cobran una gran importancia.

D. Desarrollo de rúbrica. Se desarrolla una rúbrica "ad-hoc" para esta asignatura, la cual contempla los diferentes indicadores para evaluar el nivel de dominio II. La publicación de esta rúbrica, así como la asignación de pesos numéricos al valor alcanzado por los descriptores, constituye el enfoque principal de la presente comunicación.

E. Evaluación de proyecto, desarrollo de sondeo y análisis de resultados obtenidos. Esta última fase, es crucial ya que contempla no solo la evaluación del alumno, sino que sirve de retroalimentación para la programación de las actividades y estrategias de aprendizaje a implantar en el siguiente curso. Por tanto, un análisis correcto de los resultados, así como de la opinión del alumnado, permite una retroalimentación para la mejora de las estrategias de aprendizaje de la asignatura.

\section{Resultados}

La asignatura de Ingeniería Fluidomecánica oscila entre 130 y 140 alumnos desde el curso 2015/2016 (134 alumnos en el curso 2016/2017 y 137 alumnos en el curso 2017/2018). Los alumnos se encuentra en tercer curso en semestre B y su edad oscila a su vez entre los 21 y 27 años generalmente, aunque hay casos que esta edad es superada por alumnos que acceden de módulos de formación profesional o simplemente porque han accedido más tarde a la Universidad. Por tanto, se tiene un grupo heterogéneo de alumnos con diferentes orígenes de acceso y con inquietudes distintas, así como puntos de vista, lo que da pie a una pluralidad que enriquece el debate en las cuestiones planteadas tanto por alumnos como profesor en el aula. El curso se divide en dos grupos de teoría de 68 alumnos aproximadamente y tres de prácticas repartidos en grupos de 35-42 alumnos.

\section{Resultados de aprendizaje propuestos}

El planteamiento de la actividad tiene por objeto los siguientes resultados de aprendizaje:

1) Definir la infraestructura de regulación (depósito) a partir de los condicionante de demanda, tarifa eléctrica y sistema de distribución. 
2) Determinar la curva de consigna en situaciones de bombeo directo a depósito o bombeo inyectado a la red.

3) Seleccionar las máquinas hidráulicas necesarias en número y características hidráulicas.

4) Analizar el coste energético de la explotación del sistema de acuerdo a los equipos seleccionados, tarifa y caudales demandados.

5) Introducir al alumno el uso de software específico de simulación, concretamente EPANET.

\section{Rúbrica empleada incluyendo pesos numéricos}

En la presente sección, se describe la rúbrica empleada (Tablas 1 y 2) para llevar a cabo la evaluación de la competencia transversal "Diseño y Proyecto”.

La evaluación de la actividad contiene 6 indicadores diferentes (Tablas 1 y 2), los cuales son indicados por parte del grupo de expertos de competencias transversales desde el Instituto de Ciencias de la Educación. Dentro de cada uno de estos indicadores, se han definido 13 indicadores específicos que permiten la evaluación tanto de la competencia como la calificación del proyecto.

Como novedad, la rúbrica introduce la consideración de notas numéricas a cada uno de los descriptores, así como la distribución de pesos ponderados en función de su relación con la evaluación de las competencias específicas de la asignatura. Estas ponderaciones son descritas posteriormente.

Tabla 1. Rúbrica propuesta para la asignatura Ingeniería Fluidomecánica curso 2016/2017

\begin{tabular}{|c|c|c|c|c|}
\hline \multirow{2}{*}{ INDICADORES } & \multicolumn{4}{|c|}{ DESCRIPTORES } \\
\cline { 2 - 5 } & D. No alcanzado & C. En desarrollo & B. Bien /adecuado & A. Excelente/ejemplar \\
\hline $\begin{array}{c}\text { 1.Fundamenta el } \\
\text { contexto y la } \\
\text { necesidad del } \\
\text { proyecto }\end{array}$ & $\begin{array}{c}\text { Alude a la } \\
\text { necesidad del } \\
\text { proyecto, pero no } \\
\text { la argumenta }\end{array}$ & $\begin{array}{c}\text { Justifica la necesidad } \\
\text { del proyecto en base a } \\
\text { opiniones y juicios } \\
\text { escasamente } \\
\text { contrastados y } \\
\text { fundamentados }\end{array}$ & $\begin{array}{c}\text { Fundamenta el contexto } \\
\text { y necesidad del proyecto } \\
\text { correctamente de forma } \\
\text { correcta pero no } \\
\text { completa }\end{array}$ & $\begin{array}{c}\text { Fundamenta el contexto y } \\
\text { necesidad del proyecto } \\
\text { correcta y completamente }\end{array}$ \\
$\begin{array}{c}\text { Introducción, } \\
\text { objetivos y } \\
\text { justificación }\end{array}$ & $\begin{array}{c}\text { No existe el } \\
\text { primer punto } \\
\text { a realizar, pero no } \\
\text { justifica su necesidad } \\
\text { (o lo hace atendiendo } \\
\text { a criterios } \\
\text { equivocados })\end{array}$ & $\begin{array}{c}\text { Introduce el proyecto, } \\
\text { define objetivos, pero la } \\
\text { justificación no atiende a } \\
\text { necesidades concretas }\end{array}$ & $\begin{array}{c}\text { Introduce el proyecto, define } \\
\text { claramente los objetivos y lo } \\
\text { justifica aludiendo a } \\
\text { necesidades concretas. }\end{array}$ \\
\hline $\begin{array}{c}2 . \text { Formula los } \\
\text { objetivos del } \\
\text { proyecto con } \\
\text { coherencia } \\
\text { respecto a las } \\
\text { necesidades } \\
\text { detectadas en el }\end{array}$ & $\begin{array}{c}\text { Formula objetivos } \\
\text { sin aludir a } \\
\text { necesidades }\end{array}$ & $\begin{array}{c}\text { Formula objetivos no } \\
\text { coherentes con las } \\
\text { necesidades }\end{array}$ & $\begin{array}{c}\text { Formula objetivos } \\
\text { coherentes con las } \\
\text { necesidades }\end{array}$ & $\begin{array}{c}\text { Formula objetivos coherentes } \\
\text { con las necesidades y, } \\
\text { además, son adecuados y } \\
\text { originales para la } \\
\text { transformación de la } \\
\text { situación actual atendiendo } \\
\text { las necesidades detectadas }\end{array}$ \\
\hline
\end{tabular}

(cc) EY-NC-ND 2018, Universitat Politècnica de València

Congreso IN-RED (2018) 


\begin{tabular}{|c|c|c|c|c|}
\hline contexto & & & & \\
\hline $\begin{array}{l}\text { Introducción, } \\
\text { objetivos y } \\
\text { justificación }\end{array}$ & $\begin{array}{l}\text { No existe el } \\
\text { primer punto }\end{array}$ & $\begin{array}{c}\text { Establece objetivos, } \\
\text { pero son ambiguos o } \\
\text { imprecisos }\end{array}$ & $\begin{array}{l}\text { Establece objetivos de } \\
\quad \text { forma suficiente }\end{array}$ & $\begin{array}{c}\text { Establece objetivos claros y } \\
\text { operativos }\end{array}$ \\
\hline $\begin{array}{l}\text { 3. Planifica las } \\
\text { acciones con } \\
\text { eficacia (logra } \\
\text { objetivos) }\end{array}$ & $\begin{array}{l}\text { Planifica acciones } \\
\text { no eficaces (no } \\
\text { logra gran parte } \\
\text { de los objetivos) }\end{array}$ & $\begin{array}{l}\text { Planifica acciones } \\
\text { parcialmente eficaces } \\
\text { (logra parte de los } \\
\text { objetivos) }\end{array}$ & $\begin{array}{l}\text { Planifica acciones } \\
\text { eficaces en su gran } \\
\text { mayoría (logra gran parte } \\
\text { de los objetivos) }\end{array}$ & $\begin{array}{c}\text { Planifica acciones } \\
\text { completamente eficaces } \\
\text { (todos los objetivos han sido } \\
\text { cumplidos) }\end{array}$ \\
\hline $\begin{array}{l}\text { Para cada uno de } \\
\text { los puntos }\end{array}$ & $\begin{array}{l}\text { No existe ningún } \\
\text { tipo de } \\
\text { introducción del } \\
\text { punto a } \\
\text { desarrollar }\end{array}$ & $\begin{array}{l}\text { Hace una breve } \\
\text { descripción de lo } \\
\text { contiene el punto }\end{array}$ & $\begin{array}{c}\text { Describe y justifica el } \\
\text { punto a desarrollar, pero } \\
\text { desde una perspectiva } \\
\text { académica }\end{array}$ & $\begin{array}{c}\text { Describe y justifica la } \\
\text { necesidad del punto a } \\
\text { desarrollar desde un punto } \\
\text { de vista técnico }\end{array}$ \\
\hline $\begin{array}{l}\text { 4. Planifica las } \\
\text { acciones con } \\
\text { eficiencia (usa los } \\
\text { recursos de forma } \\
\text { óptima) } \\
\end{array}$ & $\begin{array}{l}\text { No planifica } \\
\text { acciones } \\
\text { eficientes }\end{array}$ & $\begin{array}{l}\text { Planifica acciones } \\
\text { eficientes, aunque } \\
\text { visiblemente } \\
\text { mejorables }\end{array}$ & $\begin{array}{c}\text { Planifica acciones } \\
\text { eficientes, pero no todas }\end{array}$ & $\begin{array}{l}\text { Planifica de forma completa } \\
\text { acciones eficientes }\end{array}$ \\
\hline $\begin{array}{l}\text { Curva de } \\
\text { consigna }\end{array}$ & $\begin{array}{l}\text { No se realiza el } \\
\text { cálculo }\end{array}$ & $\begin{array}{l}\text { Se realiza, pero es } \\
\quad \text { incorrecto }\end{array}$ & $\begin{array}{l}\text { El resultado es correcto, } \\
\text { pero no realiza una } \\
\text { discusión del mismo }\end{array}$ & $\begin{array}{l}\text { El resultado es correcto y el } \\
\text { alumno desarrolla un } \\
\text { análisis de los resultados. }\end{array}$ \\
\hline $\begin{array}{l}\text { Definición del } \\
\text { volumen del } \\
\text { depósito }\end{array}$ & $\begin{array}{l}\text { No se realiza el } \\
\text { cálculo }\end{array}$ & $\begin{array}{l}\text { Se realiza, pero es } \\
\quad \text { incorrecto }\end{array}$ & $\begin{array}{l}\text { El resultado es correcto, } \\
\text { pero no realiza una } \\
\text { discusión del mismo }\end{array}$ & $\begin{array}{l}\text { El resultado es correcto y el } \\
\text { alumno desarrolla un } \\
\text { análisis de los resultados. }\end{array}$ \\
\hline $\begin{array}{l}\text { Selección de las } \\
\text { bombas }\end{array}$ & $\begin{array}{l}\text { No se realiza la } \\
\text { selección }\end{array}$ & $\begin{array}{l}\text { Se realiza, pero es } \\
\quad \text { incorrecta }\end{array}$ & $\begin{array}{l}\text { El resultado es correcto, } \\
\text { pero no realiza una } \\
\text { discusión del mismo }\end{array}$ & $\begin{array}{l}\text { El resultado es correcto y el } \\
\text { alumno desarrolla un } \\
\text { análisis de los resultados } \\
\text { proponiendo otros modelos o } \\
\text { soluciones }\end{array}$ \\
\hline $\begin{array}{c}\text { Selección de las } \\
\text { bombas en directo } \\
\text { a velocidad fija } \\
\text { (BVF). }\end{array}$ & $\begin{array}{l}\text { La selección de } \\
\text { las bombas en } \\
\text { directo no está } \\
\text { calculada en base } \\
\text { a la nueva curva } \\
\text { de consigna }\end{array}$ & $\begin{array}{l}\text { Se realiza, pero es } \\
\quad \text { incorrecta }\end{array}$ & $\begin{array}{c}\text { La selección llevada a } \\
\text { cabo es correcta pero no } \\
\text { está justificada }\end{array}$ & $\begin{array}{l}\text { La selección llevada a cabo } \\
\text { es correcta y el alumno lleva } \\
\text { a cabo la justificación de la } \\
\text { misma, desarrollando } \\
\text { comparaciones con otras } \\
\text { posibles soluciones }\end{array}$ \\
\hline
\end{tabular}

Tabla 2. Rúbrica propuesta para la asignatura Ingeniería Fluidomecánica curso 2016/2017

\begin{tabular}{|c|c|c|c|c|}
\hline \multirow{2}{*}{ INDICADORES } & \multicolumn{4}{|c|}{ DESCRIPTORES } \\
\hline & D. No alcanzado & C. En desarrollo & B. Bien /adecuado & A. Excelente/ejemplar \\
\hline $\begin{array}{c}\text { Análisis } \\
\text { económico }(B V F)\end{array}$ & $\begin{array}{c}\text { El alumno no } \\
\text { desarrolla el } \\
\text { análisis } \\
\text { económico de } \\
\text { explotación diario }\end{array}$ & $\begin{array}{l}\text { Desarrolla el análisis, } \\
\text { pero es incorrecto }\end{array}$ & $\begin{array}{l}\text { Desarrolla el análisis, es } \\
\text { correcto, pero no lo } \\
\text { justifica. }\end{array}$ & $\begin{array}{l}\text { Lleva a cabo una descripción } \\
\text { pormenorizada del análisis } \\
\text { económico, desarrollando } \\
\text { indicadores y comparando } \\
\text { con otras instalaciones }\end{array}$ \\
\hline $\begin{array}{c}\text { Selección de las } \\
\text { bombas en directo } \\
\text { a velocidad } \\
\text { variable }(B V V)\end{array}$ & $\begin{array}{c}\text { La selección de } \\
\text { las bombas en } \\
\text { directo no está } \\
\text { calculada en base } \\
\text { a la nueva curva } \\
\text { de consigna, } \\
\text { trabajando a }\end{array}$ & $\begin{array}{l}\text { Se realiza, pero es } \\
\text { incorrecta }\end{array}$ & $\begin{array}{c}\text { La selección llevada a } \\
\text { cabo es correcta pero no } \\
\text { está justificada }\end{array}$ & $\begin{array}{c}\text { La selección llevada a cabo } \\
\text { es correcta y el alumno lleva } \\
\text { a cabo la justificación de la } \\
\text { misma, desarrollando } \\
\text { comparaciones con otras } \\
\text { posibles soluciones }\end{array}$ \\
\hline
\end{tabular}

(c) EY-NC-ND 2018, Universitat Politècnica de València 


\begin{tabular}{|c|c|c|c|c|}
\hline & velocidad variable & & & \\
\hline $\begin{array}{c}\text { Análisis } \\
\text { económico (BVV) }\end{array}$ & $\begin{array}{c}\text { El alumno no } \\
\text { desarrolla el } \\
\text { análisis } \\
\text { económico de } \\
\text { explotación diario }\end{array}$ & $\begin{array}{l}\text { Desarrolla el análisis, } \\
\text { pero es incorrecto }\end{array}$ & $\begin{array}{l}\text { Desarrolla el análisis, es } \\
\text { correcto, pero no lo } \\
\text { justifica. }\end{array}$ & $\begin{array}{l}\text { Lleva a cabo una descripción } \\
\text { pormenorizada del análisis } \\
\text { económico, desarrollando } \\
\text { indicadores y comparando } \\
\text { con otras instalaciones }\end{array}$ \\
\hline $\begin{array}{l}\text { 5. Identifica } \\
\text { posibles riesgos } \\
\text { inherentes al } \\
\text { proyecto }\end{array}$ & $\begin{array}{l}\text { Identifica algunos } \\
\text { riesgos, pero sin } \\
\text { analizarlos. }\end{array}$ & $\begin{array}{l}\text { Identifica vagamente } \\
\text { los riesgos que pueden } \\
\text { afectar a su proyecto }\end{array}$ & $\begin{array}{l}\text { Identifica los riesgos que } \\
\text { pueden afectar a su } \\
\text { proyecto sin enfoque } \\
\text { realista o sin establecer } \\
\text { medidas para } \\
\text { subsanarlos }\end{array}$ & $\begin{array}{c}\text { Identifica, analiza y } \\
\text { cuantifica los riesgos de } \\
\text { forma realista. Los prevé } \\
\text { acertadamente y establece } \\
\text { medidas para subsanarlos }\end{array}$ \\
\hline Conclusión & $\begin{array}{l}\text { No existe } \\
\text { conclusión }\end{array}$ & $\begin{array}{l}\text { La conclusión aparece } \\
\text { únicamente como } \\
\text { último punto del } \\
\text { proyecto, sin análisis } \\
\text { ni crítica de los } \\
\text { resultados } \\
\end{array}$ & $\begin{array}{c}\text { Se analizan los } \\
\text { diferentes resultados y se } \\
\text { comparan métodos }\end{array}$ & $\begin{array}{c}\text { Se analizan los resultados, } \\
\text { comparando ambos métodos } \\
\text { e identificando ventajas e } \\
\text { inconvenientes de cada uno } \\
\text { de ellos }\end{array}$ \\
\hline $\begin{array}{c}\text { Lenguaje, formato } \\
\text { y redacción del } \\
\text { proyecto }\end{array}$ & $\begin{array}{c}\text { La presentación } \\
\text { es deficiente, la } \\
\text { redacción y el } \\
\text { lenguaje } \\
\text { utilizados no se } \\
\text { corresponden con } \\
\text { el nivel académico } \\
\text { esperado } \\
\end{array}$ & $\begin{array}{l}\text { La presentación } \\
\text { es correcta, } \\
\text { aunque } \\
\text { mejorable, el } \\
\text { lenguaje y la } \\
\text { redacción no } \\
\text { son técnicos }\end{array}$ & $\begin{array}{l}\text { La presentación, el } \\
\text { lenguaje y la redacción } \\
\text { son correctas. El } \\
\text { contenido excede o no se } \\
\text { ajusta a lo requerido }\end{array}$ & $\begin{array}{c}\text { La presentación, el lenguaje } \\
\text { y la redacción son correctas. } \\
\text { El contenido se ajusta } \\
\text { perfectamente a lo requerido } \\
\text { para explicar cada uno de los } \\
\text { puntos }\end{array}$ \\
\hline $\begin{array}{l}\text { 6. Revisa los } \\
\text { resultados } \\
\text { previstos }\end{array}$ & $\begin{array}{l}\text { No se plantea la } \\
\text { evaluación de los } \\
\text { resultados }\end{array}$ & $\begin{array}{c}\text { Se plantea que es } \\
\text { necesaria la evaluación } \\
\text { de resultados sin } \\
\text { planificar como } \\
\text { llevarla a cabo }\end{array}$ & $\begin{array}{l}\text { Planifica } \\
\text { sistemáticamente quién, } \\
\text { cuándo y cómo se } \\
\text { evaluarán los resultados }\end{array}$ & $\begin{array}{l}\text { Planifica sistemáticamente } \\
\text { quién, cuándo y cómo } \\
\text { evaluar los resultados } \\
\text { mediante indicadores } \\
\text { contrastados }\end{array}$ \\
\hline $\begin{array}{l}\text { Revisa los } \\
\text { resultados de la } \\
\text { instalación } \\
\text { propuesta } \\
\text { mediante } \\
\text { simulación en } \\
\text { EPANET }\end{array}$ & $\begin{array}{l}\text { No realiza la } \\
\text { evaluación de los } \\
\text { resultados }\end{array}$ & $\begin{array}{l}\text { Comprueba los } \\
\text { resultados, pero no los } \\
\text { analiza }\end{array}$ & $\begin{array}{l}\text { Evalúa los resultados, } \\
\text { desarrolla una } \\
\text { metodología de } \\
\text { comprobación, pero no } \\
\text { desarrolla } \\
\text { comparaciones }\end{array}$ & $\begin{array}{l}\text { Evalúa los resultados, } \\
\text { desarrolla una metodología } \\
\text { de comprobación y compara } \\
\text { con resultados entre } \\
\text { compañeros u otras } \\
\text { instalaciones o indicadores } \\
\text { existentes en la bibliografía }\end{array}$ \\
\hline
\end{tabular}

Todos los indicadores pueden presentar cuatro descriptores diferentes (No alcanzado [D], En Desarrollo [C], Adecuado [B] o Excelente [A]). Consecuente a estos descriptores, se propone una matriz de pesos y ponderaciones, considerando que el valor numérico de la nota debe de discernir en el descriptor $\mathrm{D}$, diferenciando si el alumno no lo ha realizado o si la ha realizado, pero no ha alcanzado el indicador específico. En el caso de que el alumno no lo haya desarrollado, el valor numérico es 0 , mientras si no es alcanzado el valor es 3 . El descriptor "En desarrollo, C", va ligado a un valor numérico de 5. Si el descriptor es Bien o Adecuado, la nota asignada es 7 , mientras se asigna una nota de 10 para el descriptor Excelente. Con el hecho de introducir la evaluación de las competencias específicas, a cada uno de los indicadores se les da un peso ponderado, repartidos de la siguiente forma: 
1.Fundamenta el contexto y la necesidad del proyecto; se asigna un peso del 5\%, a consecuencia que en este nivel de desarrolla, el estudiante no es capaz de interrelacionar todos los aspectos que pueden conllevar a la decisión a llevar la actuación. Este indicador contiene un solo indicador especifico.

2. Formula los objetivos del proyecto con coherencia respecto a las necesidades detectadas en el contexto; se asigna un peso del $7.5 \%$. Consecuencia de que los objetivos del proyecto son expuestos parcialmente en el enunciado, y por lo tanto, el alumno únicamente debe localizarlos y relacionarlos con la teoría impartida, para poder llevar a cabo correctamente los indicadores propuestos en el grupo 3. Solamente contiene un indicador específico.

3. Planifica las acciones con eficacia (logra objetivos); se asigna un peso del $20 \%$ ya que el alumno tiene que introducir y proponer la metodología de resolución de la actividad propuesta. Este indicador contiene un solo indicador específico.

4. Planifica las acciones con eficiencia (usa los recursos de forma óptima); se asigna un peso del $50 \%$, como consecuencia que recoge los principales indicadores específicos que están relacionados con las competencias específicas de la asignatura. Contiene 7 indicadores específicos.

5. Identifica posibles riesgos inherentes al proyecto; se considera un peso del 7.5\% como consecuencia de que el alumno debe considerar los aspectos negativos o positivos relacionados con criterios medioambientales. Este indicador contempla 2 indicadores específicos.

6.Revisa los resultados previstos; se asigna un valor del $10 \%$ ya que el alumno debe revisar, analizar y ser crítico con los resultados obtenidos, en busca de posibles resultados incoherentes, fruto del uso de software que puede llevar a incluir datos erróneos dentro de su análisis. Solamente contiene un indicador específico.

Así la expresión que determina la nota numérica de la competencia transversal y de la específica, viene definida por la siguiente expresión (1):

$$
\text { Nota }=0.05 I_{1}+0.075 I_{2}+0.20 I_{3}+0.50 \frac{\sum_{I=1}^{I=7} I_{4}}{7}+0.075 I_{5}+0.10 I_{6}
$$

Con esta expresión se permite obtener un valor numérico a partir del uso de descriptores, $\mathrm{Si}$ la nota final se encuentra entre 0 y 4.5, el resultado de aprendizaje es "No Alcanzado, D". Si el valor se encuentra entre 4.5 y 6, el indicador alcanza el resultado "En desarrollo, C". Adecuado o Bien (B) se da para notas mayores de 6 y menores de 8. Finalmente, el resultado de aprendizaje "Excelente, A" es asignado para notas mayores de 8 y 10.

\section{Participación en el curso 2016/2017}

De los 134 alumnos matriculados, 100 alumnos desarrollaron el trabajo académico propuesto. De estos 100 alumnos, 74 de ellos, alcanzaron un nivel de adquisición de la competencia superior o igual a nivel B. La correlación numérica propuesta para este curso 2017/2018 fue aplicada a este curso 2016/17. Los resultados alcanzados en la competencia 
transversal Diseño y Proyecto, los cuales muestra que de los alumnos que finalmente desarrollaron el proyecto (100), 74 alumnos alcanzaron un nivel igual o superior a B, lo que demuestra que la conjugación entre trabajo presencial y no presencial, acompañado de una tutorización en paralelo, puede contribuir a alcanzar resultados satisfactorios. Si los trabajos del año anterior, se extrapolaran a la rúbrica propuesta para este año, la nota media obtenida sería 7.76 y solamente los alumnos que no presentaron el trabajo tuvieron una nota inferior 4.5. La nota promedio para el descriptor "En Desarrollo" sería 5.22, mientras que la nota media para el nivel “Adecuado" y "Excelente” sería 7.28 y 9.12 respectivamente.

Relativos a la satisfacción del alumnado, el desarrollo de sondeos internos específicos del desarrollo de la actividad en 2016/2017 establecieron que el 62\% consideraba que el desarrollo de la actividad contribuía a la adquisición de los resultados de aprendizaje propuestos en la asignatura. El 74\% consideraba que la actividad permitía la aplicabilidad de los conceptos desarrollados.

\section{Conclusiones}

El desarrollo de la estrategias de evaluación con metodologías activas dentro de la asignatura Ingeniería Fluidomecánica, está incrementando anualmente la motivación de los alumnos hacia la materia impartida, mejorando los resultados de aprendizaje alcanzados por ellos.

La inclusión de la asignatura dentro del Proyecto de Innovación y Mejora Educativa B07 'Desarrollo de metodologías de evaluación y diseño de rúbricas de la 'CT-05. Diseño y Proyecto' aplicado al campo de la ingeniería hidráulica' desarrollado durante el curso 2016/2017, logró con éxito sentar las bases de metodologías de trabajo y consecución de los objetivos en actividades de 'Diseño y Proyecto' en su nivel de Dominio II. El hecho de estar relacionadas con otras asignaturas, concretamente Mecánica de Fluidos, impartida en segundo curso y nivel de dominio I, permite al estudiante una estrategia de actuación en cuanto al desarrollo de proyectos de infraestructuras hidráulicas. La aplicación de estas estrategias está comenzando a constatar durante el curso 2017/2018 la adquisición de resultados de aprendizaje relacionados con la aplicabilidad de metodologías que muestran el éxito de la coordinación vertical.

Aprovechando el desarrollo de las rúbricas para dicha asignatura en 2016/2017 en la evaluación de la CT-05, se han propuestos pesos numéricos a cada uno de los descriptores, con el objeto de poder evaluar numéricamente la competencia. Este desarrollo ha sido fruto de las conversaciones y reuniones mantenidas entre el PIME y la unidad de seguimiento por parte de ICE y CESPIME.

Las encuestas realizadas, muestran la satisfacción de los estudiantes en relación al desarrollo de la actividad y los contenidos teóricos de la asignatura, así como su utilidad en las competencias laborales que deberán desarrollar en un futuro. Esta satisfacción, está fuertemente relacionada con la introducción de nuevas metodologías activas docentes.

Como consecuencia del fuerte dinamismo en los últimos cursos de la asignatura Ingeniería Fluidomecánica, en cuanto a la introducción de nuevas metodologías activas resulta necesario que las mismas vayan consolidándose para descubrir las ventajas e

(cc) EY-NC-ND 2018, Universitat Politècnica de València

Congreso IN-RED (2018) 
inconvenientes, con el objeto de mejorarlas y que puedan sustituir en un futuro los actos de evaluación clásicos.

\section{Agradecimientos}

La experiencia aquí descrita se ha llevado a cabo como parte de los trabajos desarrollados en el EICE DESMAHIA "Desarrollo de metodologías activas y estrategias de evaluación aplicadas al campo de la Ingeniería Hidráulica". Los autores agradecen a la UPV, y en particular al ICE, el apoyo prestado para llevar a cabo esta iniciativa. Del mismo modo, esta comunicación está dentro del Proyecto de Innovación y Mejora Docente "Desarrollo de Metodologías de Evaluación y Diseño de Rúbricas de la CT-05 Diseño y Proyecto aplicado al campo de la Ingeniería Hidráulica (Fase II) (PIME B24)

\section{Referencias}

Benavides, G.A.M., Morales, C.E.O., 2009. Laboratorio virtual basado en la metodología de aprendizaje basado en problemas, ABP. Rev. Educ. en Ing.

DALE, E. 1969. Audiovisual methods in teaching.

MASTERS, K., 2013. Edgar Dale’s Pyramid of Learning in medical education: a literature review. Med. Teach. 35, e1584-93. doi:10.3109/0142159X.2013.800636

MoralES, P., LANDA, V., 2004. Aprendizaje basado en prob-lemas. Theoria 13, 145-157.

PÉrez-SÁnchez, Modesto; LóPez Jiménez, Petra Amparo (2017a).El debate como instrumento complementario de aprendizaje en la competencia de responsabilidad ética, medioambiental y profesional. EN Congreso Nacional de Innovación Educativa y Docencia en Red (IN-RED 2017). (683 - 695). Valencia, Spain: Editorial UPV.

Pérez-SÁnchez, Modesto; M. Isabel Pérez-SÁnchez; LóPez Jiménez, Petra Amparo (2017b). ¿Puede un conjunto de tareas evaluar la competencia "Análisis y Resolución de problemas” en una asignatura de grado?. EN Congreso Nacional de Innovación Educativa y Docencia en Red (IN-RED 2017). (1322 - 1334). Valencia, Spain: Editorial UPV.

Servicio de InNovación Educativa, 2008. Aprendizaje Basado en Problemas. Guías rápidas sobre nuevas metodologías. Universidad Politécnica de Madrid

WoOd, D., BRunER, J.S., Ross, G., 1976. The Role of tutoring in problem solving. J. Child Psychol. Psychiatry 17, 89-100. doi:10.1111/j.1469-7610.1976.tb00381.x 